\title{
Nursing Activities Score como ferramenta para avaliar a carga do trabalho de enfermagem
}

\author{
Nursing Activities Score as a tool to assess nursing work load
}

Nursing Activities Score como herramienta para evaluar la carga de trabajo de enfermería

João Victor Batista Cabral ${ }^{1}$, Maria Mariana Barros de Melo Silveira ${ }^{1}$, Adrielle Nunes de Andrade Silva ${ }^{2}$, Amanda Tavares Xavier ${ }^{3 *}$.

\section{RESUMO}

Objetivo: Descrever o uso do Nursing Activities Score (NAS) como ferramenta para avaliar a carga de trabalho de enfermagem em unidades de terapia intensiva. Métodos: Trata-se de uma revisão integrativa realizada com publicações de 2010 a 2018 nas bases de dados da Biblioteca Virtual em Saúde (BVS). Os descritores utilizados para a pesquisa foram "Unidade de Terapia Intensiva", "Taxa de Trabalho" e "Enfermagem". Resultados: Foram encontrados 18 estudos que descreveram a utilização do NAS. A maioria utilizou o método descritivo-exploratório, com nível de evidência no estrato 2 de Oxford, publicados em 2014 e oriundos do sudeste do Brasil. O NAS ainda é pouco utilizado nas UTIs brasileiras e, através de novas pesquisas, espera-se que sua implementação aumente em instituições públicas e privadas, investindo no aumento de pessoal e com o benefício de novas tecnologias. Considerações finais: Destacamos que o NAS ainda é pouco utilizado nas UTIs brasileiras, todavia constitui-se como um instrumento importante para mensurar a carga de trabalho de enfermagem na UTI, pois contempla diversas atividades realizadas e demostrou, de modo geral, que o tempo dispensado pela enfermagem é elevado, indicando sobrecarga da equipe o que pode associar-se à ocorrência de eventos adversos e de uma assistência menos efetiva.

Palavras-chave: Unidade de Terapia Intensiva, Taxa de trabalho, Enfermagem.

\begin{abstract}
Objective: To describe the use of the Nursing Activities Score (NAS) as a tool to assess the nursing workload in intensive care units. Methods: This is an integrative review carried out with publications from 2010 to 2018 in the databases of the Virtual Health Library (VHL). The descriptors used for the research were "Intensive Care Unit", "Work Rate" and "Nursing". Results: 18 studies were found that described the use of NAS. Most used the descriptive-exploratory method, with a level of evidence in stratum 2 of Oxford, published in 2014 and coming from southeastern Brazil. NAS is still little used in Brazilian ICUs and, through new research, it is expected that its implementation will increase in public and private institutions, investing in staff growth and with the benefit of new technologies. Final considerations: We highlight that the NAS is still little used in Brazilian ICUs, however it is an important instrument to measure the nursing workload in the ICU, as it includes several activities performed and demonstrated, in general, that the time spent nursing is high, indicating overload of the team, which can be associated with the occurrence of adverse events and less effective assistance.
\end{abstract}

Keyword: Intensive Care Units, Work rate, Nursing.

1 Universidade Federal de Pernambuco (UFPE), Recife - PE.

2 Centro Universitário da Vitória de Santo Antão (UNIVISA), Vitória de Santo Antão - PE.

3 Universidade de Pernambuco (UPE), Recife - PE. *E-mail: amanda-xavier@hotmail.com 


\section{RESUMEN}

Objetivo: Describir el uso del Nursing Activities Score (NAS) como herramienta para evaluar la carga de trabajo de enfermería en las unidades de cuidados intensivos. Métodos: Se trata de una revisión integradora realizada con publicaciones de 2010 a 2018 en las bases de datos de la Biblioteca Virtual en Salud (BVS). Los descriptores utilizados para la investigación fueron "Unidad de Cuidados Intensivos", "Tasa de Trabajo" y "Enfermería". Resultados: Se encontraron 18 estudios que describían el uso de NAS. La mayoría utilizó el método descriptivo-exploratorio, con un nivel de evidencia en el estrato 2 de Oxford, publicado en 2014 y proveniente del sureste de Brasil. EI NAS todavía se usa poco en las UCI brasileñas y, a través de nuevas investigaciones, se espera que su implementación aumente en las instituciones públicas y privadas, invirtiendo en el crecimiento del personal y con el beneficio de las nuevas tecnologías. Consideraciones finales: Destacamos que el NAS aún es poco utilizado en las UCl brasileñas, sin embargo, es un instrumento importante para medir la carga de trabajo de enfermería en la $\mathrm{UCl}$, ya que contempla varias actividades realizadas y demostró, en general, que el tiempo invertido la enfermería es alta, lo que indica sobrecarga del equipo, que puede estar asociada a la ocurrencia de eventos adversos y asistencia menos efectiva.

Palabra clave: Unidades de Cuidados Intensivos, Carga de trabajo, Enfermería.

\section{INTRODUÇÃO}

As unidades de terapia intensiva (UTI) admitem pacientes com diferentes condições, desde situações menos complexas até situações extremamente graves, o que implica diferentes níveis de atenção. Portanto, para garantir um atendimento de qualidade e melhorar o funcionamento do setor, é necessário identificar as particularidades de cada paciente para avaliar a carga de trabalho da equipe de enfermagem que será prestada a cada indivíduo (QUEIJO AF e PADILHA KG 2012).

Com as intensas transições pelas quais a sociedade passou, as necessidades do paciente que estão internados em unidades de terapia intensiva tornaram-se mais especializadas, em decorrência de novas tecnologias que aumentam a sobrevida. Acompanhando esse aumento, vale destacar os custos da terapia e o tempo gasto pela equipe, principalmente os profissionais de enfermagem. Nesse novo cenário, deve ser dada maior atenção não apenas ao processo saúde-doença, mas também ao cuidado humanizado dos pacientes e familiares (CONISHI RMY, et al., 2007).

Em 1974, Cullen criou o Sistema de Pontuação de Intervenção Terapêutica (TISS), que teve como objetivo medir a gravidade dos pacientes e calcular a carga de trabalho de enfermagem através de 57 intervenções, com escores de um a quatro, que relacionavam esforço e tempo. Para cada atividade de enfermagem (NUNES BK e TOMA E, 2012; CULLEN DJ, et al. 1974). Em 1983, o TISS foi atualizado para se tornar TISS-76, o qual quantificou o tempo permitido pelo pessoal médico e de enfermagem, a complexidade do paciente e o grau de dispositivos invasivos, mantendo a classificação dos grupos (I a IV) conforme a necessidade de cuidados intensivos (KEENE AR e CULLEN DJ, 1983).

Para torná-lo mais prático, construíram uma versão de 28 itens, dividida em sete categorias: atividades básicas, suporte ventilatório, cardiovascular, renal, neurológico, metabólico e intervenções específicas. 0 TISS-28 determinou que 1 ponto equivalia a 10,6 minutos de trabalho pela equipe de enfermagem, de uma pontuação total de 78 pontos (MIRANDA DR, et al., 1996). Depois de lançado e usado em todo o mundo, o TISS-28 recebeu algumas críticas por não considerar o apoio familiar e as questões administrativas como uma pontuação.

Para mensurar o trabalho de enfermagem com mais precisão, foi criado o Nursing Activities Score (NAS), que propõe quantificar a duração da assistência de enfermagem em terapia intensiva, a qual é certificada como um indicador confiável e válido, e adaptado ao idioma português (MIRANDA DR, et al. 2003; QUEIJO AF e PADILHA KG, 2012).

O NAS é dividido em sete categorias básicas: atividades básicas, suporte ventilatório, suporte cardiovascular, suporte renal, suporte neurológico, suporte metabólico e intervenções específicas. Cada 
item de uma categoria descreve uma situação, estrutura, comportamento (ou conjunto de comportamentos) que uma enfermeira aplica a um paciente e a cada item é atribuída uma pontuação. O resultado da soma das pontuações refere-se ao tempo que o paciente necessita da atenção do profissional de enfermagem nas últimas 24 horas (MIRANDA DR, et al., 2003).

A mensuração do trabalho de enfermagem permite classificar os pacientes de acordo com os cuidados necessários (NOVARETTI MCZ, et al., 2014). A carga de trabalho de enfermagem é estabelecida quando a equipe exerce suas funções no cuidado ao paciente, direta ou indiretamente. As atividades relacionadas ao paciente estão diretamente associadas ao grau de dependência do indivíduo, à gravidade do paciente, aos processos burocráticos exigidos pela instituição e pelos colegas que compõem a equipe (ALTAFIN JAM, et al., 2014).

Este estudo teve como objetivo descrever o uso do NAS como ferramenta para avaliar a carga de trabalho de enfermagem em unidades de terapia intensiva de acordo com publicações científicas brasileiras.

\section{MÉTODOS}

Este estudo baseou-se na revisão integrativa descritivo-exploratória, com abordagem qualitativa e quantitativa, realizada em seis etapas: 1- seleção da questão para a revisão; 2 - seleção dos estudos que constituirão a amostra; 3-definição das principais características que compõem a amostra; 4-análise dos resultados dos artigos; 5-interpretação dos resultados; 6-Relato da revisão, fornecendo um exame crítico dos resultados (MENDES KS, et al., 2008).

Etapa 1 - Pergunta de revisão: Como as publicações científicas descrevem o uso de NAS como ferramenta de avaliação de carga de trabalho para o pessoal de enfermagem em unidades de terapia intensiva no Brasil?

Etapa 2 - Seleção dos estudos que compuseram a amostra: A coleta de dados para a amostra foi realizada de fontes secundárias, por meio de levantamento e análise bibliográfica de publicações indexadas, realizadas de 2010 a 2018, com o objetivo de corte atualizado da amostra com publicações sobre esse assunto na Biblioteca Virtual em Saúde (BVS). Os descritores utilizados para a pesquisa foram "Unidade de Terapia Intensiva", "Carga de Trabalho" e "Enfermagem", padronizados e presentes nos Descritores em Ciências da Saúde (DeCS) com o uso do operador booleano "AND" para a associação dos descritores.

Etapa 3 - Definição das principais características da amostra: artigos publicados em português, disponíveis online gratuitamente, com texto completo, que abordam a questão da revisão, publicados e indexados na BVS, no período mencionados acima e contendo os descritores utilizados. Principalmente, os estudos incluídos continham o uso de NAS e a avaliação da carga de trabalho de enfermagem como objetivo do estudo. Os critérios de exclusão foram: artigos citados como resenhas, notícias, teses, dissertações, arquivos, editoriais, capítulos de livros, aqueles que não se enquadram no prazo estabelecido e estudos em unidades de neonatologia e pediatria, por serem unidades diferenciadas em relação ao perfil do paciente, sua comparação com o ambiente do paciente adulto se tornaria difícil e tendenciosa.

Etapa 4 - Análise dos resultados dos artigos: análise descritiva após leitura crítica dos resumos de cada artigo e sua subsequente leitura completa. Caso consistente com o tópico proposto, ou seja, resultados que denotassem a carga de trabalho da enfermagem em unidades de terapia intensiva brasileiras representadas pelo uso do NAS, o artigo foi incluído como amostra. A avaliação dos estudos em relação ao nível de evidência (NE) foi norteada pelas determinações do Medicina Baseada em Evidências do Oxford Center. Foram encontrados 55 artigos, dos quais 18 foram selecionados como amostra (Quadro 1).

Etapa 5 - Interpretação dos resultados: para expressar, interpretar e analisar as evidências dos artigos, foram elaboradas tabelas descritivas (quadros 2 e 3 ) que resumem as evidências dos artigos encontrados para responder à pergunta da revisão, utilizando pontos descritivos. O quadro 2 contém: nível de evidência / ano, título do artigo, base de dados / periódico, autor(es) e tipo de estudo e o quadro 3 contém o título do estudo e seus resultados. 
Etapa 6 - Relato de revisão: foi classificado em tópicos, de acordo com as evidências apresentadas pela amostra, para permitir uma construção descritiva que responda à questão norteadora.

Quadro 1 - Níveis de evidência de acordo com o tipo de estudo de acordo com o medicamento baseado em evidências do Oxford Center.

\begin{tabular}{|c|c|}
\hline NE & TIPO DE ESTUDO \\
\hline $1 \mathrm{~A}$ & $\begin{array}{l}\text { Revisão sistemática de ensaios clínicos controlados randomizados. Ensaios clínicos } \\
\text { randomizados bem delineados com resultados clínicos relevantes. }\end{array}$ \\
\hline 1B & Ensaio clínico controlado randomizado com intervalo de confiança estreito. \\
\hline $1 \mathrm{C}$ & Resultados terapêuticos do tipo "Tudo ou nada". Estudo de \\
\hline $2 \mathrm{~A}$ & Série de casos controlados. \\
\hline $2 B$ & $\begin{array}{l}\text { Revisão sistemática homogênea de estudos de coorte (com grupos de comparação e } \\
\text { controle de variáveis). }\end{array}$ \\
\hline $2 \mathrm{C}$ & $\begin{array}{c}\text { Estudo de coorte com baixa qualidade de randomização, controle ou sem acompanhamento } \\
\text { prolongado, estudo de coorte transversal. }\end{array}$ \\
\hline $3 A$ & Resultados da pesquisa (observação de resultados terapêuticos ou evolução clínica). \\
\hline $3 B$ & Revisão sistemática homogênea de estudos de caso com um grupo de controle. \\
\hline 4 & Casos práticos com um grupo de controle. \\
\hline 5 & Relatos de casos e séries sem definição de controle de casos. \\
\hline
\end{tabular}

Fonte: Centre for Evidence-Based Medicine, 2009. Dados coletados por Cabral JVB, et al., 2020.

\section{RESULTADOS}

Foram selecionados 18 artigos, dos quais apenas dois adotaram o método de revisão, os demais utilizaram o estudo descritivo-exploratório da abordagem qualitativa, utilizando o formulário para coleta do NAS. Os artigos de revisão foram incluídos por se tratar de uma revisão da literatura sobre o estado da arte, justificando sua inclusão ao denotar que o tema foi abordado em estudos que buscavam o uso de NAS por meio de outras publicações (Quadro 2).

Quanto ao nível de evidência, verificou-se que a maioria dos estudos foi classificada no estrato 2, com 1 artigo 2A, 7 2B e 9 2C, que classificou os estudos com nível moderado de evidência. Apenas um estudo foi classificado no estrato 5 . Os artigos predominantes foram encontrados na base de dados LILACS Literatura em Ciências da Saúde da América Latina e no Caribe, com nove publicações, seguidos por sete artigos encontrados no MEDLINE - Sistema de análise e recuperação de literatura médica on-line e dois no Bases de dados de enfermagem - BDENF.

A Revista Brasileira de Enfermagem apresentou três publicações sobre o assunto, seguida pela Acta Paulista de Enfermagem, Escola de Enfermagem da USP, Revista Anna Nery de Enfermagem, Revista Latino-Americana de Enfermagem e Revista Brasileira de Terapia Intensiva, com duas publicações cada. Os demais, Jornal Eletrônico de Enfermagem, Cogitare Nursing, Revista RENE (Rede de Enfermagem do Nordeste), Journal of Science, Care and Health contribuíram com uma publicação.

Em 2014, houve o maior número de publicações, com um total de cinco artigos, seguido por 2017 com quatro artigos. Em 2011, 2012, 2015 e 2016, foram encontrados dois artigos em cada um e, em 2013, apenas um artigo.

Com relação ao tema dos artigos, cinco deles relacionaram NAS a eventos adversos que ocorrem em unidades de terapia intensiva, como infecções, quedas e lesões por pressão. Dois desses correlacionaram a carga de trabalho de enfermagem com infecções e eventos. Um artigo relaciona a carga de trabalho com pacientes com IRA (lesão renal aguda) e outro com IMC (índice de massa corporal). Os demais não encontraram relação, mas observou-se que a gravidade dos pacientes, as comorbidades, o sexo e o tempo de permanência exercem maior influência nos eventos adversos que ocorrem nos locais estudados (Quadro 3). 
Quadro 2 - Amostra do estudo: descrição dos estudos de acordo com o nível de evidência, título, ano, banco de dados / periódico, autor (es) e tipo de estudo.

\begin{tabular}{|c|c|c|c|c|}
\hline $\begin{array}{l}\text { Nivel de } \\
\text { Evidência / } \\
\text { Ano }\end{array}$ & Título & Bases de Dados/Periódico & Autores & Tipo de Estudo \\
\hline 2C / 2011 & $\begin{array}{c}\text { Nursing Activities Scores (NAS): Carga de trabalho de } \\
\text { enfermagem em UTI e fatores associados }\end{array}$ & $\begin{array}{l}\text { BDENF/ Revista Ciência, Cuidado e } \\
\text { Saúde }\end{array}$ & Inoue KC, Kuroda CM, Matsuda LM & Descritivo Exploratório \\
\hline $2 \mathrm{C} / 2011$ & $\begin{array}{c}\text { Carga de trabalho de enfermagem em Unidade de Terapia } \\
\text { Intensiva de um hospital de ensino }\end{array}$ & LILACS/ Acta Paul Enfermagem & Panunto MR, Guirardello EB & Descritivo \\
\hline 2C / 2012 & $\begin{array}{c}\text { Sítios assistenciais em unidade de terapia intensiva e } \\
\text { relação do Nursing Activities Score com a infecção } \\
\text { hospitalar }\end{array}$ & $\begin{array}{l}\text { LILACS/ Escola Anna Nery Revista } \\
\text { de Enfermagem }\end{array}$ & Cyrino CMS, Dell'Acqua MCQ & $\begin{array}{l}\text { Descritivo Transversal } \\
\text { Quantitativo }\end{array}$ \\
\hline 2B / 2013 & $\begin{array}{c}\text { Carga de trabalho de enfermagem em unidade de terapia } \\
\text { intensiva públicas e privadas }\end{array}$ & $\begin{array}{c}\text { LILACS/ Revista Brasileira de Terapia } \\
\text { Intensiva }\end{array}$ & $\begin{array}{c}\text { Nogueira LS, Koike KM, Sardinha DS, } \\
\text { et al. }\end{array}$ & Coorte Retrospectiva \\
\hline 2B / 2014 & $\begin{array}{l}\text { Sobrecarga de trabalho da Enfermagem e incidentes e } \\
\text { eventos adversos em pacientes internados em UTI }\end{array}$ & $\begin{array}{l}\text { MEDLINE/ Revista Brasileira de } \\
\text { Enfermagem }\end{array}$ & Novaretti MCZ, et al. & $\begin{array}{l}\text { Coorte Prospectiva } \\
\text { Qualitativa }\end{array}$ \\
\hline 5 / 2014 & $\begin{array}{l}\text { Dimensionamento de enfermagem em Unidade de Terapia } \\
\text { Intensiva: evidências sobre o Nursing Activities Score }\end{array}$ & BDENF/ Revista RENE & $\begin{array}{l}\text { Ferreira PC, Machado RC, Vitor AF, } \\
\text { Lira ALBC, Martins QCS }\end{array}$ & Revisão Integrativa \\
\hline 2B / 2014 & $\begin{array}{c}\text { Nursing Activities Score e a carga de trabalho em unidade } \\
\text { de terapia intensiva de um hospital universitário }\end{array}$ & $\begin{array}{l}\text { LILACS/ Revista Brasileira de Terapia } \\
\text { Intensiva }\end{array}$ & $\begin{array}{c}\text { Altafin JA, Grion CM, Tanita MT, Festti } \\
\text { J, Cardoso LT, Veiga CF, et al }\end{array}$ & Longitudinal Prospectivo \\
\hline $2 C / 2014$ & $\begin{array}{c}\text { Carga de trabalho de enfermagem em uma unidade de } \\
\text { terapia intensiva de trauma }\end{array}$ & $\begin{array}{l}\text { LILACS/ Revista Eletrônica de } \\
\text { Enfermagem }\end{array}$ & $\begin{array}{l}\text { Goulart LL, Aoki RN, Vegian CFL, } \\
\text { Guirardello EB }\end{array}$ & Descritivo \\
\hline 2C / 2014 & $\begin{array}{l}\text { Nursing Activities Score: Carga de trabalho de } \\
\text { enfermagem em unidade de queimados }\end{array}$ & $\begin{array}{l}\text { MEDLINE/ Revista Latino-Americana } \\
\text { de Enfermagem }\end{array}$ & $\begin{array}{c}\text { Camuci MB, Martins JT, Cardeli AAM, } \\
\text { Robazzi MLCC }\end{array}$ & $\begin{array}{l}\text { Exploratório Descritivo } \\
\text { Quantitativo }\end{array}$ \\
\hline 2B / 2015 & $\begin{array}{l}\text { Carga de trabalho de Enfermagem: preditor de infecção } \\
\text { relacionada à assistência de saúde na terapia intensiva }\end{array}$ & $\begin{array}{l}\text { MEDLINE/ Revista da Escola de } \\
\text { Enfermagem da USP }\end{array}$ & $\begin{array}{l}\text { Nogueira LS, Ferretti-Rebustini REL, } \\
\text { Poveda VB, et al. }\end{array}$ & Coorte Retrospectiva \\
\hline 2B / 2015 & $\begin{array}{c}\text { Correlação entre carga de trabalho de enfermagem e } \\
\text { gravidade dos pacientes críticos gerais, neurológicos e } \\
\text { cardiológicos }\end{array}$ & $\begin{array}{l}\text { LILACS/ Escola Anna Nery Revista } \\
\text { de Enfermagem }\end{array}$ & $\begin{array}{l}\text { Siqueira EMP, Ribeiro MD, Souza } \\
\text { RCS, et al. }\end{array}$ & Coorte Prospectiva \\
\hline $2 A / 2016$ & $\begin{array}{l}\text { Carga de trabalho de enfermagem e ocorrência de eventos } \\
\text { adversos na terapia intensiva: revisão sistemática }\end{array}$ & $\begin{array}{l}\text { MEDLINE/ Revista Escola de } \\
\text { Enfermagem da USP }\end{array}$ & Oliveira AC, Garcia PC, Nogueira LS. & Revisão Sistemática \\
\hline 2C / 2016 & $\begin{array}{l}\text { Impacto financeiro do quadro de profissionais de } \\
\text { enfermagem requerido em unidade de terapia intensiva }\end{array}$ & $\begin{array}{c}\text { MEDLINE/ Revista Latino-Americano } \\
\text { de Enfermagem }\end{array}$ & $\begin{array}{l}\text { Araújo TR, Menegueti MG, Auxiliadora- } \\
\text { Martins M, Castilho V, et al. }\end{array}$ & Descritivo Quantitativo \\
\hline 2B / 2017 & Nursing Activities Score e a lesão renal aguda & $\begin{array}{c}\text { MEDLINE/ } \\
\text { Revista Brasileira de Enfermagem }\end{array}$ & Coelho, FUA, et al. & $\begin{array}{l}\text { Coorte Retrospectiva } \\
\text { Quantitativa }\end{array}$ \\
\hline 2B / 2017 & $\begin{array}{c}\text { Gravidade e carga de trabalho relacionadas a eventos } \\
\text { adversos em UTI }\end{array}$ & $\begin{array}{c}\text { MEDLINE/ Revista Brasileira de } \\
\text { Enfermagem }\end{array}$ & Serafim CTR, et al. & Estudo Prospectivo \\
\hline 2C / 2017 & $\begin{array}{c}\text { Carga de trabalho de enfermagem relacionada ao índice } \\
\text { de massa corporal de pacientes críticos }\end{array}$ & LILACS/ Acta Paul Enfermagem & Goulart LL, et al. & Descritivo Quantitativo \\
\hline $2 \mathrm{C} / 2017$ & $\begin{array}{c}\text { Dimensionamento de pessoal de enfermagem na UTI- } \\
\text { adulto de hospital universitário público }\end{array}$ & LILACS/ Cogitare Enfermagem & Borges $\mathrm{F}$, et al. & Transversal Quantitativo \\
\hline
\end{tabular}

Fonte: Cabral JVB, et al., 2020. 
Quadro 3 - Amostra do estudo: descrição dos estudos de acordo com o Título e os Resultados.

\begin{tabular}{|c|c|}
\hline Título & Resultados \\
\hline $\begin{array}{l}\text { Nursing Activities Scores (NAS): Carga de trabalho de } \\
\text { enfermagem em UTI e fatores associados }\end{array}$ & $\begin{array}{l}\text { A carga de trabalho de enfermagem é próxima ao valor referente à alta carga e ainda é } \\
\text { necessário repensar a dinâmica da admissão de pacientes nesse serviço, pois, dentre as } \\
\text { variáveis investigadas, o tratamento cirurgia é o único que interfere diretamente no aumento } \\
\text { da carga de trabalho. }\end{array}$ \\
\hline $\begin{array}{l}\text { Carga de trabalho de enfermagem em Unidade de Terapia } \\
\text { Intensiva de um hospital de ensino }\end{array}$ & $\begin{array}{l}\text { O NAS constitui um instrumento importante para mensurar a carga de trabalho de } \\
\text { enfermagem na UTI, pois contempla diversas atividades de enfermagem realizadas. } \\
\text { Ressalta-se a importância de tornar sua aplicação parte da rotina diária do enfermeiro. }\end{array}$ \\
\hline $\begin{array}{l}\text { Sítios assistenciais em unidade de terapia intensiva e relação do } \\
\text { Nursing Activities Score com a infecção hospitalar }\end{array}$ & $\begin{array}{l}\text { O NAS médio foi de } 71,72 \% \text {. Em relação à infecção hospitalar antes e após o implante, } \\
\text { houve redução nas taxas de pneumonia, no entanto, a carga de trabalho de enfermagem } \\
\text { permaneceu constante. }\end{array}$ \\
\hline $\begin{array}{l}\text { Nursing Activities Score e demanda de trabalho de enfermagem } \\
\text { em terapia intensiva }\end{array}$ & $\begin{array}{l}\text { Em relação à carga de trabalho de enfermagem, o escore médio total foi de } 68,1 \% \text {, o que } \\
\text { corresponde ao percentual de tempo gasto pelo profissional de enfermagem no atendimento } \\
\text { direto ao paciente em } 24 \text { horas. Os resultados mostraram que os pacientes tinham uma alta } \\
\text { necessidade de cuidados. }\end{array}$ \\
\hline $\begin{array}{l}\text { Carga de trabalho de enfermagem em unidade de terapia } \\
\text { intensiva públicas e privadas }\end{array}$ & $\begin{array}{l}\text { O NAS médio calculado na admissão em unidades de terapia intensiva foi de } 61,9 \text { e, na alta, } \\
\text { de } 52,8 \text {. Pacientes de instituições públicas apresentaram maior carga de trabalho na } \\
\text { admissão na UTI, mas até a alta houve um declínio mais pronunciado na demanda por } \\
\text { atendimento entre os sobreviventes internados na unidade. }\end{array}$ \\
\hline $\begin{array}{l}\text { Sobrecarga de trabalho da Enfermagem e incidentes e eventos } \\
\text { adversos em pacientes internados em UTI }\end{array}$ & $\begin{array}{c}\text { Evidenciou-se que } 78 \% \text { dos incidentes sem lesões e eventos adversos em pacientes } \\
\text { estavam relacionados à esfera de enfermagem, aumentando os dias de internação com a } \\
\text { associação do risco de mortalidade. }\end{array}$ \\
\hline $\begin{array}{l}\text { Dimensionamento de enfermagem em Unidade de Terapia } \\
\text { Intensiva: evidências sobre o Nursing Activities Score }\end{array}$ & $\begin{array}{c}\text { Considera-se que o NAS foi utilizado de maneira correta, embora não amplamente divulgada } \\
\text { no Brasil, com média em todas as obras superior a } 50 \% \text {, sendo incompatível com o } \\
\text { preconizado pelo Ministério da Saúde. }\end{array}$ \\
\hline $\begin{array}{l}\text { Nursing Activities Score e a carga de trabalho em unidade de } \\
\text { terapia intensiva de um hospital universitário }\end{array}$ & $\begin{array}{l}\text { Existe uma alta carga de trabalho de enfermagem no hospital de pesquisa, associada ao tipo } \\
\text { de hospitalização (cirurgia de emergência) e ao desfecho do paciente (não sobrevivente). A } \\
\text { gravidade do paciente e as disfunções orgânicas mostraram correlação com a carga de } \\
\text { trabalho de enfermagem. }\end{array}$ \\
\hline $\begin{array}{c}\text { Carga de trabalho de enfermagem em uma unidade de terapia } \\
\text { intensiva de trauma }\end{array}$ & $\begin{array}{c}\text { Alta carga de trabalho, sem diferenciar o período de internação e alta. Houve correlação com } \\
\text { o NAS e o APACHE II. }\end{array}$ \\
\hline
\end{tabular}




\begin{tabular}{|c|c|}
\hline Título & Resultados \\
\hline $\begin{array}{l}\text { Nursing Activities Score: Carga de trabalho de enfermagem em } \\
\text { unidade de queimados }\end{array}$ & $\begin{array}{l}\text { Identificou-se que há sobrecarga de trabalho da equipe de enfermagem na UTI queimada da } \\
\text { unidade investigada. }\end{array}$ \\
\hline $\begin{array}{l}\text { Carga de trabalho de Enfermagem: preditor de infecção } \\
\text { relacionada à assistência de saúde na terapia intensiva }\end{array}$ & $\begin{array}{l}\text { Fatores preditivos para infecções relacionadas à saúde em pacientes hospitalizados na UTI } \\
\text { analisada foram: tempo de permanência na unidade, gravidade, sexo masculino e } \\
\text { comorbidades. A carga de trabalho não influenciou a ocorrência desse resultado. }\end{array}$ \\
\hline $\begin{array}{l}\text { Correlação entre carga de trabalho de enfermagem e gravidade } \\
\text { dos pacientes críticos gerais, neurológicos e cardiológicos }\end{array}$ & $\begin{array}{l}\text { Houve uma correlação moderada entre a gravidade do paciente neurológico e a carga de } \\
\text { trabalho de enfermagem, e o NAS e o SAPS3 foram utilizados como escores. }\end{array}$ \\
\hline $\begin{array}{l}\text { Carga de trabalho de enfermagem e ocorrência de eventos } \\
\text { adversos na terapia intensiva: revisão sistemática }\end{array}$ & $\begin{array}{l}\text { A influência da carga de trabalho de enfermagem requerida por um paciente na UTI foi } \\
\text { identificada como fator de risco para o aparecimento de eventos adversos devido a } \\
\text { infecções, lesões por pressão ou erros de medicação nos estudos de } 6 \text { de agosto } \\
\text { analisados. }\end{array}$ \\
\hline $\begin{array}{l}\text { Impacto financeiro do quadro de profissionais de enfermagem } \\
\text { requerido em unidade de terapia intensiva2 }\end{array}$ & $\begin{array}{l}\text { O NAS foi relevante na identificação das horas trabalhadas de acordo com a gravidade do } \\
\text { paciente e na identificação dos custos da assistência de enfermagem na UTI, gerando um } \\
\text { orçamento real para a adaptação do número quantitativo de profissionais de enfermagem. }\end{array}$ \\
\hline Nursing Activities Score e a lesão renal aguda & $\begin{array}{l}\text { O NAS de pacientes com LRA foi maior em comparação com pessoas que não } \\
\text { desenvolveram LRA. O NAS aumentou com o agravamento da LRA, confirmando uma maior } \\
\text { necessidade de cuidados de enfermagem entre esses pacientes. }\end{array}$ \\
\hline $\begin{array}{l}\text { Gravidade e carga de trabalho relacionadas a eventos adversos } \\
\text { em UTI }\end{array}$ & $\begin{array}{c}\text { Este estudo demonstrou que a incidência de eventos adversos está diretamente relacionada } \\
\text { ao tempo de internação e à gravidade do paciente. A relação entre a incidência de eventos } \\
\text { adversos e a carga de trabalho de enfermagem não apresentou relação estatisticamente } \\
\text { significante. }\end{array}$ \\
\hline $\begin{array}{l}\text { Carga de trabalho de enfermagem relacionada ao índice de } \\
\text { massa corporal de pacientes críticos }\end{array}$ & $\begin{array}{l}\text { Os resultados deste estudo permitiram concluir que a carga de trabalho de enfermagem na } \\
\text { UTI, segundo o NAS, não diferiu entre os grupos com diferentes IMCs. }\end{array}$ \\
\hline $\begin{array}{c}\text { Dimensionamento de pessoal de enfermagem na UTI-adulto de } \\
\text { hospital universitário público }\end{array}$ & $\begin{array}{c}\text { Concluiu-se que o déficit dos profissionais de enfermagem era alto e isso estava relacionado } \\
\text { ao fato de a carga de trabalho da Unidade ser alta, o que, dada a gravidade clínica da } \\
\text { clientela e a requisitos profissionais, que atribuíram uma ampla aplicação de cuidados } \\
\text { Profissionais com maior nível de treinamento. }\end{array}$ \\
\hline
\end{tabular}

Fonte: Cabral JVB, et al., 2020. 
A maioria dos estudos foi realizada na região sudeste do Brasil, destacando o estado de São Paulo com doze publicações, seguido pelo estado do Paraná com quatro. Os estados do Rio Grande do Norte e Piauí, no nordeste do Brasil, contribuíram com uma publicação. Dos 18 artigos publicados, apenas um indica o impacto financeiro da instituição, investindo na adequação do número de profissionais de enfermagem. Os outros usam o NAS associado ao Simplified Acute Physiology Score (SAPS 3), Acute Physiologic and Chronic Health Evaluation (APACHE II) e Sequential Organ Failure Assessment Score (SOFA) ou realizam a revisão bibliográfica dos artigos que utilizaram o NAS para obtenção de médias de carga de trabalho do profissional de enfermagem da unidade de terapia intensiva.

\section{DISCUSSÃO}

Considerando que o NAS foi criado em 2002 e as publicações brasileiras se materializaram de forma mais evidente a partir de 2006, supõe-se que nesse período houve de modo construtivo um processo de familiarização com o novo escore entre os profissionais e pesquisadores para implementação na realidade das unidades de terapia intensiva brasileiras, obtendo resultados diferentes das descobertas europeias. Destaca-se que no Brasil, o TISS-28 é o instrumento mais frequentemente utilizado para mensuração da carga de trabalho da enfermagem, assim como a complexidade do paciente. Apesar de haver similaridade entre os instrumentos e de ambos serem de fácil uso, o processo de iniciação do uso de novos escores é difícil por questões de familiaridade e fatores culturais (INOUE KC, et al. 2011).

Em um dos estudos, através da análise de artigos publicados entre 2002 e 2010, pode-se observar que o NAS médio permaneceu elevado, com valores acima de $50 \%$, o que indica uma sobrecarga nas atividades dos profissionais de enfermagem na unidade de terapia intensiva (FERREIRA PC, et al,. 2015). O escore do NAS representa o tempo de trabalho que o paciente crítico necessitou de um profissional de enfermagem em 24 horas, o que é alcançado através do percentual de tempo dedicado pela equipe ao atendimento, que varia de zero a $176,8 \%$. Se o escore for igual a $100 \%$, significa que o paciente precisa de $100 \%$ do tempo de atendimento do profissional de enfermagem em 24 horas. Cada ponto da pontuação é equivalente a 14,1 minutos do tempo dispensado para a execução dos cuidados de enfermagem (QUEIJO AF e PADILHA KG, 2012).

O NAS mínimo encontrado nesta pesquisa foi de $61,9 \%$ no estudo de Oliveira AC, et al. (2016), enquanto o máximo, de $104 \%$ foi identificado nos resultados de Inoue KC et al. (2011), ambos representando um quadro de excesso de trabalho da enfermagem, seja pela complexidade dos pacientes ou pelo subdimensionamento do pessoal. Quando é realizado o cálculo equivalente ao escore em horas trabalhadas, observa-se que o escore mínimo é de 14, 5 horas de dedicação ao paciente na UTI. O máximo é equivalente a 24,4 horas de dedicação ao paciente, ou seja, não seria possível prestar cuidados adequados e seguros e de acordo com a Resolução do Conselho Federal de Enfermagem (COFEN) ํo 543/2017 (BRASIL, 2017).

Quanto ao dimensionamento adequado, alguns artigos fizeram referência à Portaria $n^{\circ}$ 3432, de 12 de agosto de 1998 do Ministério da Saúde (BRASIL, 1998), que estabelece que deve haver um técnico para cada dois pacientes, o que ainda é vigente com atualização da Resolução de Diretoria Colegiada ํㅡ 07 de Fevereiro de 2010, que estabelece os critérios mínimos para o funcionamento das unidade de terapia intensiva no Brasil (ALTAFIN JAM, et al., 2014; FERREIRA PC, et al., 2014; GOULART LL, et al., 2014; SIQUEIRA EMP, et al., 2015; ARAUJO TR, et al., 2016). Além disso, também houve citação da Resolução COFEN 293/2004, que considerou, para fins de dimensionamento, 17,9 horas de assistência de enfermagem prestada por pacientes críticos, revogada pela Resolução COFEN no 543/2017 (INOUE KC, et al., 2011; PANUNTO MR e GUIRARDELLO EB, 2012; FERREIRA PC, et al., 2014; SIQUEIRA EMP, et al., 2015).

Os resultados demonstraram uma alta carga de trabalho em todas as unidades de terapia intensiva estudadas, uma vez que se verificou uma pontuação média superior a $66,5 \%$ do tempo que o profissional de enfermagem dedica ao paciente. Em um dos estudos concluiu-se que os pacientes cirúrgicos precisavam de mais atenção da equipe do que os clínicos e que a média do NAS era de 104\%, demonstrando que um 
profissional não podia prestar cuidados adequados e seguros a um único paciente. A crescente demanda por cuidados intensivos no Brasil e o acesso às diversas tecnologias e terapias de substituição vital são fatores que explicam o aumento da gravidade dos pacientes e assim do tempo dispensado pela enfermagem (INOUE KC, et al., 2011).

Em um estudo publicado em 2013, foi comparada a carga de trabalho da equipe de enfermagem em UTIs públicas e privadas. Fatores sociais, o atraso na admissão do paciente na UTI e o déficit no leito do Sistema Único de Saúde (SUS) tornam o paciente usuário do SUS mais grave, motivo pelo qual se tem os maiores escores de gravidade durante a internação em comparação com serviços privados. Entretanto, durante o período de internação até a alta, há basicamente um equilíbrio no escore de ambos os serviços. Os serviços privados, de modo geral, possuem protocolos de risco, que permitem a identificação de pacientes potencialmente graves e assim sua admissão precoce na UTI, mesmo que sua gravidade não seja elevada, o que reduz a necessidade de intervenções de enfermagem e assim contribuem para a diferença com os pacientes do sistema único (NOGUEIRA LS, et al., 2013).

Dois estudos publicados em 2014 investigaram a associação do NAS elevado com os índices de gravidade SOFA e APACHE II, que revelaram associação significativa não apenas com a gravidade do paciente e com os procedimentos realizados, mas também com problemas administrativos e organizacionais de uma UTI. Em um estudo, foi feita uma comparação entre o TISS 28 e o NAS e concluiuse que o TISS 28 apenas mensura o trabalho em contato direto com o paciente, enquanto o NAS abrange outras atividades rotineiras da UTI, como apoio emocional às famílias. O NAS, deste modo, surge como uma ferramenta valiosa para avaliar, de maneira global, o trabalho da enfermagem na terapia intensiva, haja vista que a atuação da equipe, especialmente do enfermeiro, transcende a rotina de cuidados diretos ao paciente, incluindo atividades gerenciais, administrativas e de educação em saúde (ALTAFIN JAM, et al., 2014; GOULART LL, et al., 2014).

Outro estudo avaliou a relação entre o NAS e o SAPS 3, um sistema prognóstico desenvolvido para estimar a mortalidade hospitalar por meio de variáveis demográficas e fisiológicas e o diagnóstico de admissão na UTI. Os resultados mostram que a gravidade e a carga de trabalho foram maiores nos grupos de pacientes clínicos e cirúrgicos gerais em comparação aos pacientes neurológicos e cardíacos (SIQUEIRA EMP, et al. 2015). Em estudo realizado em 2017, observou-se que pacientes diagnosticados com lesão renal aguda em UTI geral necessitam de mais cuidados do que pacientes sem esse diagnóstico, obtendo um NAS de 45,9\% e 40,7\%, respectivamente. Assim, maior é a carga de trabalho de enfermagem necessária para a manutenção adequada desses indivíduos. (COELHO FUA, et al., 2017).

Diferentes estudos compararam e destacaram que a distribuição adequada dos profissionais de enfermagem aumenta a segurança do paciente, além de mitigar e prevenir efeitos adversos e complicações como infecções, lesões por pressão, extubação, perda acidental de dispositivos e mortalidade em geral (CYRINO CMS e DELL'ACQUA MCQ, 2012; FERREIRA, et al., 2014; NOGUEIRA LS, et al., 2015; OLIVEIRA AC, et al.,2016; SERAFIM CTR, et al.,2017). Destaca-se que o adequado dimensionamento da equipe de enfermagem é fator já conhecido como um elemento fundamental para o alcance da efetividade do cuidado. Não se pode pensar em cuidado complexo prestado ao paciente grave, sem que haja equipe de enfermagem. Outro ponto importante é o fato de que a segurança do paciente é assunto crescente e vem motivando um movimento dinâmico no país, que em sua maioria, é protagonizado pela enfermagem.

Com as dificuldades no controle de custos em saúde, principalmente em Unidades de Terapia Intensiva é fundamental o desenvolvimento de estudos que busquem o entendimento da relação custo-benefício no provimento efetivo e essencial do pessoal de enfermagem. Apenas um artigo investigou o impacto financeiro em uma instituição privada na região sul do Brasil, calculando que o aumento da equipe de enfermagem de acordo com o NAS médio (86,6\%) seria em média $30 \%$, estimando-se um valor mensal de 15 mil reais, equivalente a aproximadamente $42,59 \%$ do orçamento da instituição. O NAS mostrou- se efetivo enquanto instrumento no processo de identificação de custos relacionado à assistência de enfermagem em unidade de terapia intensiva, fornecendo, deste modo, subsídios aos gestores e administradores para planejamento e aplicação orçamentária a curto e longo prazos (ARAUJO TR, et al., 2016). 


\section{CONSIDERAÇÕES FINAIS}

Apesar da expansão da terapia intensiva no Brasil e do incremento em relação ao número e gravidade dos pacientes e das novas tecnologias, o que implica diretamente no aumento do trabalho da enfermagem, as publicações sobre a temática ainda são reduzidas, mesmo na vigência da resolução 543/2017 do COFEN, que normatiza o assunto e deve assim, ser elemento motivador de reflexão sobre a necessidade de estudos que permitam compreender os impactos dos diversos fatores na dinâmica de trabalho na UTI. Destacamos que o NAS ainda é pouco utilizado nas UTIs brasileiras, todavia constitui-se como um instrumento importante para mensurar a carga de trabalho de enfermagem na UTI.

\section{REFERÊNCIAS}

1. ALTAFIN JAM, et al. Nursing Activities Score and workload in the intensive care unit of a university hospital. Rev Bras Ter Inten, 2014; 26: 292-298.

2. ARAUJO TR, et al. Impacto financeiro do quadro de profissionais de enfermagem requerido em unidade de terapia intensiva. Rev Latino-Am Enf. 2016; 24: 1-9.

3. BORGES F, et al. Dimensionamiento del personal de enfermería en la UCl adulta del Hospital Universitario Público. Código de Enfermagem, 2017; 22: 01-08.

4. BRASIL. Ministério da Saúde (MS). Portaria $n^{\circ}$ 3.432, de 12 de agosto de 1998. Brasília: MS; 1998. Disponível em: http://bvsms.saude.gov.br/bvs/saudelegis/gm/1998/prt3432_12_08_1998.html.

5. CONSELHO FEDERAL DE ENFERMAGEM (COFEN). Resolução Cofen 543/2017.Brasília: COFEN; 2017. Disponível em: http://www.cofen.gov.br/resolucao-cofen-5432017_51440.html.

6. CAMUCI MB, et al. Nursing Activities Score: Carga de trabalho de enfermagem em unidade de queimados. Rev Latino-Am Enf. $2014 ; 22: 325-31$.

7. CENTRE FOR EVIDENCE-BASED MEDICINE (CEBM). Oxford Centre for Evidence-Based Medicine: Levels of Evidence (March 2009). Oxford: CBEM; 2009. Disponível em: http://www.cebm.et/oxfordcentre-evidence-basedmedicine-levels-evidencemarch-2009.

8. COELHO FUA, et al. Nursing Activities Score e a lesão renal aguda. Rev Bras Enferm, 2017; 70: 475-780.

9. CULLEN DJ, et al. Therapeutic intervention scoring systema method for quantitative comparison of patient care. Crit Care Med, 1974; 2 : 57-60.

10. CYRINO CMS, DELL'ACQUA MCQ. Sitios de asistencia en la unidad de cuidados intensivos y relación de la Puntuación de Actividades de Enfermería con infección hospitalaria. Rev Esc Anna Nery, 2012; 16: 712-718.

11. FERREIRA PC, et al. Dimensionamento de enfermagem em Unidade de Terapia Intensiva: evidências sobre 0 Nursing Activities Score. Rev Rene, 2014; 15: 888-97.

12. GOULART LL, et al. Carga de trabalho de enfermagem em uma unidade de terapia intensiva de trauma. Rev Elet de ENF, 2014; $16: 346-351$.

13. INOUE KC, et al. Nursing Activities Scores (NAS): Carga de trabalho de enfermagem em UTI e fatores associados. Rev Cienc Cuid Salud, 2011; 10:134-140.

14. KEENE AR, CULLEN DJ. Therapeutic Intervention Scoring System: update. Crit Care Med, 1983; 11 1-3.

15. LEITE IRL, et al. Nursing Activities Score e demanda de trabalho de enfermagem em terapia intensiva. Acta Paul Enf, 2012; 25: 1-7.

16. MIRANDA DR, et al. Simplified Therapeutic Intervention Scoring System: The TISS-28 items--Results from a multicenter study. Crit Care Med, 1996; 24: 64-73.

17. MIRANDA DR, et al. Nursing activities score. Crit Care Med, 2003; 31:374-82.

18. MENDES KS, et al. Revisão integrativa: método de pesquisa para a incorporação de evidências na saúde e na enfermagem. Tex Cont Enf, 2008; 17: 758-764.

19. NOGUEIRA LS, et al. Carga de trabalho de Enfermagem: preditor de infecção relacionada à assistência de saúde na terapia intensiva. Rev Esc Enf USP, 2015; 49: 36-42.

20. NOVARETTI MCZ, et al. Sobrecarga de trabalho da Enfermagem e incidentes e eventos adversos em pacientes internados em UTI. Rev Bras Enf, 2014; 67: 692-699.

21. NUNES BK, TOMA E. Dimensionamento de pessoal de enfermagem de uma unidade neonatal: utilização do Nursing Activities Score. Rev Latino-Am. Enf, 2013; 21:348-355.

22. OLIVEIRA AC, et al. Carga de trabalho de enfermagem e ocorrência de eventos adversos na terapia intensiva: revisão sistemática. Rev Esc Enf USP, 2016; 50:683-694.

23. PANUNTO MR, GUIRARDELLO EB. Carga de trabalho de enfermagem em Unidade de Terapia Intensiva de um hospital de ensino. Acta Paul Enf, 2012; 25:96-101.

24. PANUNTO MR, GUIRARDELLO EB. Carga de trabalho de enfermagem em Unidade de Terapia Intensiva de um hospital de ensino. Acta Paul Enf, 2012; 25:96-101.

25. QUEIJO AF, PADILHA KG. Nursing Activities Score (NAS): adaptação transcultural e validação para a língua portuguesa. Rev Esc Enf USP, 2009; 43:1018-1025.

26. SIQUEIRA EMP, et al. Correlação entre carga de trabalho de enfermagem e gravidade dos pacientes críticos gerais, neurológicos e cardiológicos. Rev Esc Anna Nery, 2015; 19:233-238. 